\title{
Introduction to Mineral Exploration
}

EDITED BY

ANTHONY M. EVANS

WITH CONTRIBUTIONS FROM

WILLIAM L. BARRETT

TIMOTHY BELL

ANTHONY M. EVANS

JOHN MILSOM

CHARLES J. MOON

BARRY C. SCOTT

MICHAEL K.G. WHATELEY

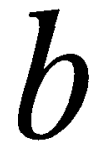




\section{Contents}

Contributors viii

Preface

Units and Abbreviations

\section{Part 1-Principles}

1 Ore, Mineral Economics and Mineral Exploration

1.1 Introduction

1.2 Mineral Economics

1.3 Important Factors in the Economic Recovery of Minerals

1.4 Some Factors Governing the Choice of Exploration Areas

1.5 Rationale of Mineral Exploration

1.6 Further Reading

2 The Mineralogy of Economic Deposits

2.1 Introduction

2.2 Mineralogical Investigations

2.3 Further Reading

\section{Mineral Deposit Geology}

29

3.1 Nature and Morphology of

Orebodies

3.2 Wall Rock Alteration

3.3 Plate Tectonics and the Global

Distribution of Ore Deposits

3.4 Further Reading

3.5 Summary of Chapters 1, 2 and 3

\section{Reconnaissance} Exploration

4.1 Exploration Planning 44

4.2 Desk Studies and Reconnaissance

4.3 Summary

4.4 Further Reading

54

62

62

\section{$5 \quad$ From Prospect to Predevelopment}

5.1 Finding a Drilling Target
5.2 When to Drill and When to Stop 77

5.3 Recycling Prospects 82

5.4 Summary 90

5.5 Further Reading 90

\section{$6 \quad$ Remote Sensing and} Photogeology

6.1 Introduction 92

6.2 The Landsat System 92

6.3 SPOT 102

6.4 Photogeology 103

6.5 Summary 112

6.6 Further Reading 112

\section{$7 \quad$ Geophysical Methods $\quad \mathbf{1 1 3}$}

7.1 Introduction 113

7.2 Airborne Surveys 113

7.3 Magnetic Surveys $\quad 116$

7.4 Gravity Method 119

$\begin{array}{ll}7.5 & \text { Radiometrics } \\ 7.6 & 120\end{array}$

$\begin{array}{lll}7.6 & \text { Resistivity } & 122\end{array}$

7.7 Spontaneous Polarization (SP) 124

7.8 Induced Polarization (IP) 124

7.9 Electromagnetic Methods $\quad 126$

7.10 Transient Electromagnetics (TEM) 128

7.11 VLF and Magnetotellurics $\quad 129$

7.12 Seismic Methods 130

7.13 Borehole Geophysics 132

7.14 Exploration Programmes 132

7.15 Integration of Geological and Geophysical Data

7.16 The Role of the Geologist in Geophysical Exploration

7.17 Negotiations with Contractors 136

7.18 Further Reading 137

8 Exploration Geochemistry 138

8.1 Planning 138

8.2 Analysis 142

8.3 Interpretation 144

8.4 Reconnaissance Techniques $\quad 149$ 
vi CONTENTS

$\begin{array}{llr}8.5 & \text { Follow-up Sampling } & 159 \\ 8.6 & \text { Summary } & 160 \\ 8.7 & \text { Further Reading } & 160 \\ & & \\ 9 & \text { Evaluation Techniques } & 161 \\ 9.1 & \text { Sampling } & 161 \\ 9.2 & \text { Pitting and Trenching } & 176 \\ 9.3 & \text { Auger Drilling } & 176 \\ 9.4 & \text { Other Drilling } & 177 \\ 9.5 & \text { Mineral Resource and } & \\ & \text { Ore Reserve Estimation, } & \\ & \text { Grade Calculations } & 186 \\ 9.6 & \text { Geotechnical Data } & 193 \\ 9.7 & \text { Summary } & 199 \\ 9.8 & \text { Further Reading } & 200 \\ 9.9 & \text { Appendix } & 200 \\ & & \\ 10 & \text { Feasibility Studies } & 203 \\ 10.1 & \text { Introduction } & 203 \\ 10.2 & \text { Value of Mineralization } & 203 \\ 10.3 & \text { Valuation of Mineralization } & 208 \\ 10.4 & \text { Mineral Project Valuation and } & \\ & \text { Selection Criteria } & 211 \\ 10.5 & \text { Risk } & 214 \\ 10.6 & \text { Inflation } & 215 \\ 10.7 & \text { Mineral Project Finance } & 217 \\ 10.8 & \text { Summary } & 220 \\ 10.9 & \text { Further Reading } & 220 \\ & & \end{array}$

\section{PART II-Case Studies}

11 Cliffe Hill Quarry, LeicestershireDevelopment of Aggregate Reserves 223

11.1 Introduction 223

11.2 Phase 1-Preliminary Desk Study

11.3 Phase 2 - Initial Follow-up

$$
\text { Work }
$$

11.4 Phase 3 - Detailed Follow-up Work 225

11.5 Phase 4-Project Implementation 231

11.6 Summary 232

12 Soma Lignite Basin, Turkey

12.1 Introduction

12.2 Exploration Programmes

12.3 Geology

238

12.4 Data Assessment

241

12.5 Geotechnical Investigation

12.6 Lignite Quality

246

248

12.7 Lignite Reserve Estimates 252

12.8 Surface Mine Evaluation 254

12.9 Summary 257

12.10 Further Reading 257

13 Witwatersrand

Conglomerate GoldWest Rand

258

13.1 Introduction 258

13.2 Geology 258

13.3 Economic Background 267

13.4 Exploration Models 270

13.5 Exploration Methods 272

13.6 Reserve Estimation 280

13.7 Exploration and Development of the Southern Part of the West

Rand Goldfield

281

13.8 Conclusions

290

14 A Volcanic-associated Massive Sulphide Deposit -Kidd Creek, Ontario

14.1 Introduction 292

14.2 Volcanic-associated Massive Sulphide Deposits 292

14.3 An Exploration Model for VMS Deposits

14.4 The Kidd Creek Mine 304

14.5 Conclusions 315

15 Disseminated Precious Metals-Trinity Mine, Nevada

15.1 Background 316

15.2 Trinity Mine, Nevada 320

15.3 Exploration 320

15.4 The Geology of Pershing County and the Trinity District

323

15.5 Development and Mining 329

15.6 Mineral Processing 329

15.7 Environmental Considerations 330

15.8 Grade Estimation 331

15.9 Ore Reserve Estimation 339

15.10 Summary and Conclusions 343 
CONTENTS vii

16 Narrow Vein DepositsWheal Jane, Cornwall

344

16.1 Introduction

16.2 Economic Background

16.3 Geology

16.4 Exploration Programmes in Cornwall and Devon

16.5 Wheal Jane Exploration

16.6 Production History
16.7 Underground Exploration

361

16.8 Mining

361

16.9 Mineral Processing

16.10 The Environmental Impact of Mine Closure

16.11 Summary

References Index

365

366 